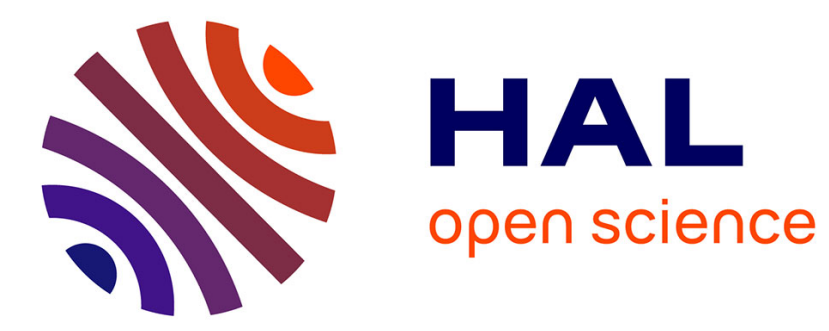

\title{
Surface-wave pyroelectric photorefractive solitons
}

J. Safioui, E. Fazio, F. Devaux, M. Chauvet

\section{To cite this version:}

J. Safioui, E. Fazio, F. Devaux, M. Chauvet. Surface-wave pyroelectric photorefractive solitons. Optics Letters, 2010, 35 (8), pp.1254-1256. 10.1364/OL.35.001254 . hal-00476869

\section{HAL Id: hal-00476869 \\ https://hal.science/hal-00476869}

Submitted on 9 Apr 2021

HAL is a multi-disciplinary open access archive for the deposit and dissemination of scientific research documents, whether they are published or not. The documents may come from teaching and research institutions in France or abroad, or from public or private research centers.
L'archive ouverte pluridisciplinaire HAL, est destinée au dépôt et à la diffusion de documents scientifiques de niveau recherche, publiés ou non, émanant des établissements d'enseignement et de recherche français ou étrangers, des laboratoires publics ou privés. 


\title{
Surface-wave pyroelectric photorefractive solitons
}

\author{
Jassem Safioui, ${ }^{1}$ Eugenio Fazio, ${ }^{2}$ Fabrice Devaux,${ }^{1}$ and Mathieu Chauvet ${ }^{1, *}$ \\ ${ }^{1}$ FEMTO-ST, UMR 6174, Université de Franche-Comté, 16 Route de Gray, 25000 Besançon, France \\ ${ }^{2}$ Ultrafast Photonics Laboratory, Dipartimento di Energetica and CNISM, Sapienza Università Roma, \\ via Scarpa 16, 00161 Roma, Italy \\ *Corresponding author: mathieu.chauvet@univ-fcomte.fr
}

\begin{abstract}
Surface-wave solitons, induced by the pyroelectric effect, are formed at the interface between a photorefractive ferroelectric medium and a linear medium. These optical solitons are trapped in both transverse dimensions and are efficiently attracted to the interface. The asymmetric shape of the nonlinear index change formed under the charge saturation regime is responsible for the surface-wave solitons' formation. Experimental demonstrations are performed in a lithium niobate sample with moderate temperature change. The phenomenon is successfully explained through numerical simulations.
\end{abstract}

Since the first theoretical study of nonlinear optical surface waves [1,2], they have attracted a lot of attention from the scientific community. These selftrapped waves are formed at the interface between two media with at least medium having nonlinear optical properties. Most demonstrations concern a selfconfined wave whose energy is, for the most part, located in a nonlinear medium of higher average refractive index than the adjacent medium. Trapping at the interface occurs thanks to a balance between the repelling force due to the presence of the nearby low-index medium and the self-formation of an asymmetric index profile that pushes the beam toward the surface. When a local nonlinearity is used, such as the Kerr effect, the asymmetric index profile forms because the beam is distorted as a result of the nearby interface. For such a pure local nonlinear effect the beam thus has to be launched in the vicinity of the interface to give rise to a surface wave. As an example, discrete surface solitons (SSs) have recently been demonstrated at the boundary of photonic lattices in a Kerr medium [3,4] and in a quasi-local photorefractive (PR) medium [5]. In contrast, a nonlocal nonlinearity, for which the refractive index perturbation extends away from the beam, offers an easier way to form surface waves. Indeed, light can be attracted to the surface from far away to finally form a surface wave. Such a nonlocal SS has recently been formed at a bulk-dielectric interface by using a thermal nonlinearity [6]. Surface waves can also be observed by using a nonlocal PR nonlinearity when charge diffusion is dominant $[7,8]$, which can even lead to formation of SSs when an additional strong electrical bias is applied [9].

In this Letter we present the formation of SSs that belong to the latter category. They are formed by use of the pyroelectric effect in a PR medium. The SS is efficiently trapped in two transverse dimensions while it travels few micrometers under the sample surface. Experimental demonstrations are performed in a photonic grade $\mathrm{LiNbO}_{3}$ crystal [10].

The recent discovery of pyroelectric spatial solitons [11] in a PR medium, so-called pyrolitons, has opened up new possibilities. The use of the pyroelectric effect also provides an optimized arrangement to propagate a wave at the surface with very low loss, since no electrodes are present at the interface. Considering the very large soliton bending observed in bulk strontium barium niobate (SBN) [12] and $\mathrm{LiNbO}_{3}$ [13], it constitutes a favorable configuration to observe SSs. Indeed, such a beam lateral displacement is the sign of an asymmetric induced index profile that can efficiently constrain a beam against an interface. This asymmetric index profile is attributed to higher-order space charge field components [14] induced by a saturation of charges involved in the PR effect. The underlying physics was successfully exposed in the framework of a numerical model for $\mathrm{LiNbO}_{3}$ [15].

To show that these characteristics are suitable to trap a SS, numerical 2D simulations have been developed first.

To model beam self-trapping in pyroelectric PR crystal we consider a time-dependent band-transport model with a single deep trap. Electrons are displaced by the photovoltaic effect and under drift current along the crystal $c$ axis while charge diffusion is neglected. An open-circuit crystal at homogeneous and steady temperature is considered.

When illuminated with a light intensity distribution $I(x, y)$, the evolution of the charge density $\rho$ is given by

$$
\frac{\partial \rho}{\partial t}=-\mu e \vec{\nabla}(N \cdot \vec{E})-\beta_{\mathrm{ph}} \vec{\nabla}\left[\left(N_{d}-N_{d}^{+}\right) I\right] \cdot \vec{c}
$$

where the free electron density $N$, ionized donor density $N_{d}^{+}$, and total internal electric field $\vec{E}$ are given by Eqs. (2)-(4), respectively:

$$
\begin{gathered}
N=\frac{s\left(I+I_{d}\right)\left(N_{d}-N_{d}^{+}\right)}{\gamma N_{d}^{+}}, \\
N_{d}^{+}=N_{a}+\frac{\rho}{e},
\end{gathered}
$$




$$
\vec{E}(\vec{r})=E_{\mathrm{py}} \cdot \vec{c}+\frac{1}{4 \pi \epsilon_{0} \epsilon_{r}} \iiint_{V} \rho\left(\vec{r}^{\prime}\right) \frac{\vec{r}-\vec{r}^{\prime}}{\left|\vec{r}-\vec{r}^{\prime}\right|^{3}} \mathrm{~d} V .
$$

$N_{d}$ is the total donor density; $N_{a}$ is the density of ionized shallow acceptors. $I_{d}=\beta / s$ is the equivalent dark irradiance with $\beta$ and $s$ the thermal and photoexcitation coefficients, $\gamma$ is the recombination coefficient, $\mu$ is the electron mobility, $\beta_{\mathrm{ph}}$ is the photovoltaic coefficient, and $e$ is electron charge.

The pyroelectric field $E_{\mathrm{py}}$ due to the spontaneous polarization change is given by

$$
E_{\mathrm{py}}=\Delta E=-\frac{1}{\epsilon_{0} \epsilon_{r}} p \Delta T .
$$

where $\epsilon_{0}$ and $\epsilon_{r}$ are the vacuum and relative dielectric constants, respectively, $p$ is the pyroelectric coefficient, and $\Delta T$ is the temperature variation relative to equilibrium room temperature. Any temperature change induces a spontaneous polarization variation and thus gives rise to an internal electric field $E_{\mathrm{py}}$.

The electric field evolution is obtained by solving iteratively, in the two transverse dimensions $x$ and $y$, the set of equations (1)-(4) [15] in conjunction with the wave propagation equation. Starting from initial conditions, the electron density is calculated from Eq. (2), and space charge variation after a time step $\Delta t$ is deduced from Eq. (1). Then the ionized donor density is obtained from Eq. (3), and the electric field is deduced from Eq. (4). The inferred 2D refractive index perturbation $\Delta n$ due to the linear electro-optic effect is finally used to calculate light propagation along the $z$ axis in the perturbed medium by a split-step Fourier transform method according to the nonlinear propagation equation (6):

$$
\left\{\frac{\partial}{\partial z}-\frac{i}{2 k} \Delta_{\perp}\right\} A=i k \Delta n A,
$$

where $\Delta_{\perp}=\partial^{2} / \partial x^{2}+\partial^{2} / \partial y^{2}$ is the transverse operator, $A$ is the slowly varying amplitude of the light field, and $k$ is the wavenumber in the medium. To successfully solve the propagation equation the weak optical field that is present in air is truncated. Neglecting this evanescent field is acceptable, since the beam propagates at grazing incidence along a steep index interface.

A numerical simulation showing the formation of a $\mathrm{SS}$ is presented in Fig. 1. Initial PR parameters are $N_{d}=2 \times 10^{21} \mathrm{~m}^{-3}, N_{a}=N_{d}^{+}=0.99 N_{d}, N=0$; other parameters are similar to those in [15]. A $17 \mu \mathrm{m} \mathrm{FWHM}$ $532 \mathrm{~nm}$ focused beam is launched about $60 \mu \mathrm{m}$ below the $\mathrm{LiNbO}_{3}$ sample edge and propagates with a slight angle toward the $\mathrm{LiNbO}_{3}$ air interface [Fig. 1(a)]. When the beam hits the interface, interference fringes are formed over a large area [Fig. 1(b)], because the beam has diffracted along its propagation in this initial linear regime. As the PR effect develops, beam self-focusing occurs, and fringes start to vanish [Figs. 1(c) and 1(d)]. In a more advanced state the beam sticks to the interface and propagates unchanged owing to an asymmetric induced index dis-

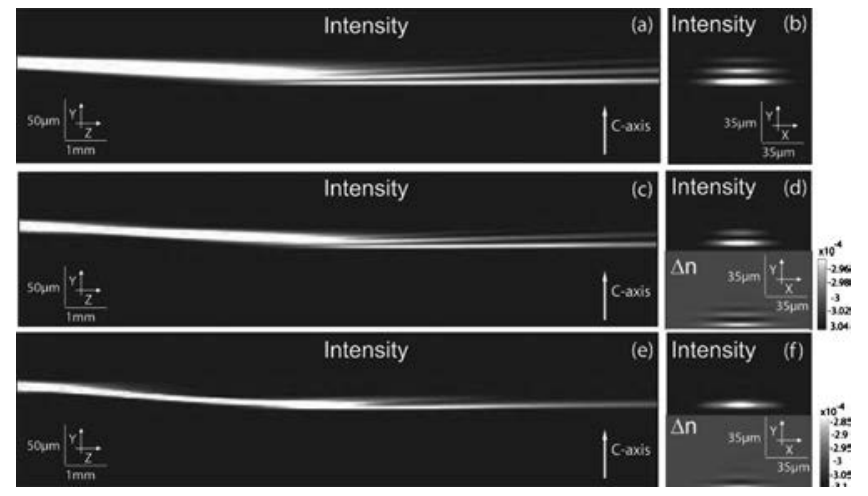

Fig. 1. Numerical simulation showing the formation of a SS in $\mathrm{LiNbO}_{3}$ by using pyroelectricity. Left column, sideview intensity distribution of the beam; right column, corresponding view at the exit face along with refractive index distribution $\Delta n$ at three characteristic times. $\Delta T=20^{\circ} \mathrm{C}$ and initial PR parameters $N_{d}=2 \times 10^{21} \mathrm{~m}^{-3}, \quad N_{a}=N_{d}^{+}$ $=0.99 N_{d}, N=0$.

tribution [Figs. 1(e) and 1(f)]. Such a simulation, with an initial tilted beam, reveals that the nonlinear selfbending is able to counteract total reflection. Simulations performed with a beam injected parallel to the interface also form a stable SS. Because the initial ratio $N_{d} / N_{a}$ is slightly larger than unity, saturation of the deep center occurs when light is present, which finally induces an asymmetric space charge field and index distribution [15]. Note that trapping against the $+c$ interface is not possible, since both beam bending and the repelling effect occur in the same direction, which pushes the beam away from the interface.

To experimentally demonstrate the pyrolelectric SS, 20-mm-long $\mathrm{LiNbO}_{3}$ samples are cut from a 0.5 -mm-thick $c$-cut photonic grade wafer. A $532 \mathrm{~nm}$ extraordinary polarized beam is focused to a $13 \mu \mathrm{m}$ (FWHM) spot at the entrance of the sample and propagates in the $20-\mathrm{mm}$-long direction. A Peltier element is used to control the sample temperature with stability better than $0.1^{\circ} \mathrm{C}$. Input and output faces of the crystal are observed on a CCD camera via imaging lenses.

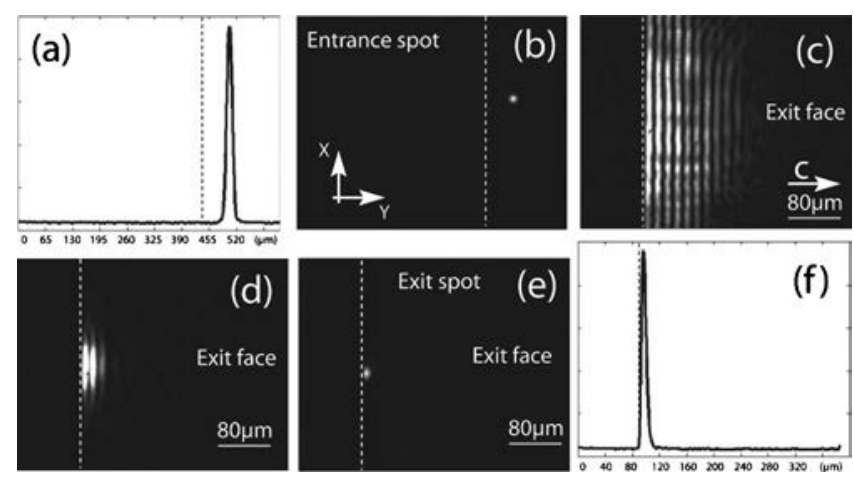

Fig. 2. Experimental demonstration of a pyroelectric SS in a 2-cm-long $\mathrm{LiNbO}_{3}$ sample. (a) Beam intensity profile along the $c$ axis and (b) distribution at the entrance face of the sample. (c), (d) Evolution of output beam intensity once sample temperature is set to $40^{\circ} \mathrm{C}$; (e), (f) formed soliton and its profile. The dashed line indicates the interface location. 


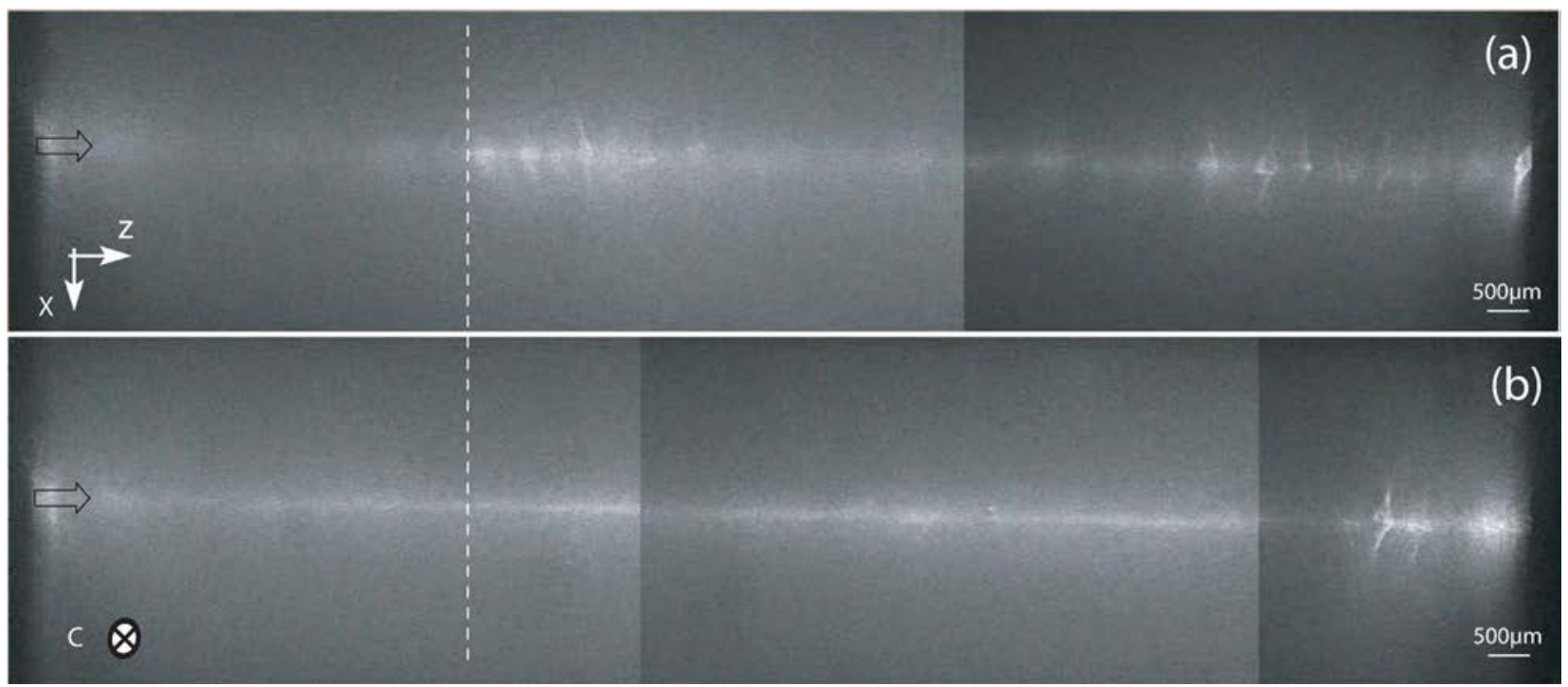

Fig. 3. (Color online) Side view of (a) a beam launched inside a $\mathrm{LiNbO}_{3}$ sample in diffraction regime and (b) when a pyroelectric SS is formed. The dashed line is beam reflection location in the linear regime. Views are constructed from several images.

The launched beam intensity profile and distribution are depicted in Figs. 2(a) and 2(b). This beam is injected about $60 \mu \mathrm{m}$ under the $-c$ interface and travels with a small angle toward this face. Total reflection occurs in the middle of the sample and forms Lloyd fringes in a large region [Fig. 2(c)]. When the crystal temperature is raised from room temperature $\left(20^{\circ} \mathrm{C}\right)$ to $40^{\circ} \mathrm{C}$ the interference fringes gradually disappear as the PR effect builds up [Fig. 2(d)]. This self-trapping is due to the screening of the internal pyroelectric field as depicted in [11]. Two minutes after the temperature change this $80 \mu \mathrm{W}$ beam exits in an optimally confined SS [Fig. 2(e)]. The beam FWHM is $7 \mu \mathrm{m}$ along the $c$ axis and $13 \mu \mathrm{m}$ perpendicular to the $c$ axis. It is trapped against the interface as revealed by the asymmetric beam profile along the $c$ axis [Fig. 2(f)]. A steeper intensity change is present at the interface because of the large index difference between air and $\mathrm{LiNbO}_{3}$. An additional experiment has been performed to confirm that, once formed, the SS sticks to the interface. A $\mathrm{LiNbO}_{3}$ sample with an unpolished $-c$ face is used, and observations are made with a low-magnification optical system from above this surface. These observations rely on light scattering along the unpolished interface. When a $100 \mu \mathrm{W}$ focused beam (15 $\mu \mathrm{m}$ FWHM) is launched $80 \mu \mathrm{m}$ below the $-c$ face, no scattered light is first detected, while after about $5 \mathrm{~mm}$ propagation distance scattering is observed because some light reaches the interface as a result of beam diffraction [Fig. 3(a)]. When the crystal temperature is raised to $40^{\circ} \mathrm{C}$, a SS is formed about $2 \mathrm{~mm}$ after the entrance face as shown by the narrow line of scattered light observed up to the exit face [Fig. 3(b)].

In conclusion we have shown that a 2D SS can be formed at the interface between a low-index medium and a medium with an optical nonlinearity that gives rise to an asymmetric index distribution. The experimental demonstration is performed in a PR pyroelectric $\mathrm{LiNbO}_{3}$ crystal where the nonlinearity is trig- gered by a $20^{\circ} \mathrm{C}$ temperature increase. Efficient light attraction and localization underneath the surface is due to charge saturation as corroborated with a numerical model.

E. Fazio is grateful to the Université de FrancheComté for the visiting professorship under which part of this work has been performed.

\section{References}

1. W. J. Tomlinson, Opt. Lett. 5, 323 (1980).

2. D. Mihalache, M. Bertolotti, and C. Sibilia, Prog. Opt. 27, 229 (1989).

3. K. G. Makris, S. Suntsov, D. N. Christodoulides, G. Stegeman, and A. Haché, Opt. Lett. 30, 2466 (2005).

4. S. Suntsov, K. G. Makris, D. N. Christodoulides, G. I. Stegeman, A. Haché, R. Morandotti, H. Yang, G. Salamo, and M. Sorel, Phys. Rev. Lett. 96, 063902 (2006).

5. X. Wang, A. Bezryadina, Z. Chen, K. G. Makris, D. N. Christodoulides, and G. I. Stegeman, Phys. Rev. Lett. 98, 123903 (2007).

6. B. Alfassi, C. Rotschild, O. Manela, M. Segev, and D. N. Christodoulides, Phys. Rev. Lett. 98, 213901 (2007).

7. M. Cronin-Golomb, Opt. Lett. 20, 2075 (1995).

8. G. S. Garcia Quirino, J. J. Sanchez-Mondragon, and S. Stepanov, Phys. Rev. A 51, 1571 (1995).

9. H. Z. Kang, T. H. Zhang, B. H. Wang, C. B. Lou, B. G. Zhu, H. H. Ma, S. M. Liu, J. G. Tian, and J. J. Xu, Opt. Lett. 34, 3298 (2009).

10. E. Fazio, F. Renzi, R. Rinaldi, M. Bertolotti, M. Chauvet, W. Ramadan, A. Petris, and V. I. Vlad, Appl. Phys. Lett. 85, 2193 (2004).

11. J. Safioui, F. Devaux, and M. Chauvet, Opt. Express 17, 22209 (2009).

12. Shih, P. Leach, M. Segev, M. H. Garrett, G. Salamo, and G. C. Valley, Opt. Lett. 21, 324 (1996).

13. M. Chauvet, V. Coda, H. Maillotte, E. Fazio, and G. Salamo, Opt. Lett. 30, 1977 (2005).

14. S. R. Singh, M. I. Carvalho, and D. N. Christodoulides, Opt. Commun. 130, 288 (1996).

15. F. Devaux, V. Coda, M. Chauvet, and R. Passier, J. Opt. Soc. Am. B 25, 1081 (2008). 